\title{
Towards a Theory of Limited Indeterminism in Branching Space-times
}

\author{
Thomas Müller
}

Received: 28 June 2009 / Accepted: 22 August 2009 / Published online: 22 June 2010

(C) The Author(s) 2010. This article is published with open access at Springerlink.com

\begin{abstract}
Branching space-times (BST; Belnap, Synthese 92:385-434, 1992) is the most advanced formal framework for representing indeterminism. BST is however based on continuous partial orderings, while our natural way of describing indeterministic scenarios may be called discrete. This paper establishes a theorem providing a discrete data format for BST: it is proved that a discrete representation of indeterministic scenarios leading to BST models is possible in an important subclass of cases. This result enables the representation of limited indeterminism in BST and hopefully paves the way for the representation of substances with capacities in that framework.
\end{abstract}

Keywords Modality • Indeterminism • Agency

Nuel Belnap's Branching Space-Times (BST; [1]) is the most advanced formal framework for indeterminism that is out there: it combines successfully the notion of an open future with a relativistic view of space and time. Historically, BST grew out of considerations of agency in the stit ("seeing to it that") framework: ${ }^{1}$ the relativistic notion of space-like separation was meant to provide an objective basis for the "independence of different agents' simultaneous choices" postulate, which in branching-time (BT) based stit theory appeared as a deus ex machina. ${ }^{2}$ After much technical development of both BST and

\footnotetext{
${ }^{1}$ Cf. Belnap et al. [8] for a full overview. The earliest paper on the theory is Belnap and Perloff [7]. ${ }^{2}$ I'd like to thank several participants of the Nuelfest, April 2009, for helpful remarks on the early history of BST.
}

T. Müller $(\varangle)$

Department of Philosophy, Utrecht University, Heidelberglaan 6,

3584 CS Utrecht, The Netherlands

e-mail: Thomas.Mueller@phil.uu.nl 
stit theory separately, Belnap himself has made the link between the two explicit in one of his most recent papers [6], once again proving the benefit of perseverance in formal philosophical work.

On a somewhat broader and less technical level, he has also described the key motivation behind BST as providing a framework for indeterminism that is "equally proto-scientific and proto-humanistic" [5, p. 19].The theory should thus not be limited to what Sellars [24] called the "manifest image" of man (including, e.g., our concept of agency), nor to what he called the "scientific image" (including, e.g., relativity theory). ${ }^{3}$ Rather, by staying at a formal level the theory should lend itself to applications in both of the great theoretical endeavors in which we engage: coming to grips with the world in which we live in terms growing out of our lived experience, as well as in terms provided by science. In bridging that gap, BST would help to establish the "humanistic" and the scientific respectability of the concept of indeterminism.

This approach is superior to most if not all other approaches to the determinism/indeterminism issue out there: Remaining formal and neutral about the manifest/scientific image divide is to be preferred above a "Lebenswelt only" ideology and, perhaps more importantly since that is the option more commonly chosen, above a scientistic excommunication of Lebenswelt concepts. The latter approach often goes together with an unargued but strong conviction that being precise necessitates being a determinist; so establishing indeterminism as scientifically respectable with formal rigor is especially important.

\section{On BST's Status as Both Proto-scientific and Proto-humanistic}

For a theory to be proto-scientific and proto-humanistic, that theory must preclude neither the findings of empirical science nor central concepts of our Lebenswelt. ${ }^{4}$ Belnap honors these constraints by providing a sophisticated formal framework that, in contradistinction to earlier approaches to indeterminism, is not at odds with relativity theory, ${ }^{5}$ and which provides elbow room for a substantive theory of indeterminism in action. The theory remains proto-

\footnotetext{
${ }^{3}$ By employing Sellars's terminology I am not claiming that Belnap endorses the dichotomy as strict.

${ }^{4}$ Arguably such a theory may nibble away some concepts on both sides. Probably at any historical stage of development there's bad science as well as superstition. But the dialectical starting point has to be science and life as we find them, phlogiston, witches and all. Argument is needed to weed out anything on either side, and the historical course of events testifies to that.-Note that by "science" I mean empirical science, not free-floating theorizing. Thus, e.g., at the present stage of development I do not think that any constraints flow forth from the several approaches to quantum gravity, except for the fact that there are both quantum effects and gravitational effects in our world.

${ }^{5}$ It is known that there is a remaining problem concerning some models of general relativity theory. But the theory is compatible with many models of the general theory and with all models of the special theory, which is a great achievement.
} 
scientific by not implementing any actual scientific claims (in fact, even the instruments for representing such claims are left open), and it remains protohumanistic in not implementing any Lebenswelt concepts (such as action, or in fact even plain things-substances-and their properties).

The abstractness of BST is an advantage that comes, one could say, with a set of promissory notes: BST's status as a proto-theory invites complementation, and in both directions. The challenge for such complementation is to proceed in such a way as to maintain the original balance, or rather impartiality, between scientific and manifest image: to build forth on something that would leave us and the world whole.

Since the publication of the original BST article in 1992, some progress has been made towards the implementation of scientific concepts in BST, especially with respect to an understanding of quantum theory within BST. ${ }^{6}$ The implementation of Lebenswelt concepts has however remained a recalcitrant problem, despite Belnap's success at applying BST to agency mentioned above. Part of the reason for that may be the following: While BST is not a physical theory, it shares many structural properties of current space-time theories (while going significantly beyond them in representing modality as well). Such theories are, to use an image employed by Butterfield [10], pointilliste, or atomistic: BST's most basic notion is that of a "possible point-event", much as space-time theories build upon a notion of (actual) point event. Now from a Lebenswelt perspective, points may be (idealized) locations of things, but they aren't things themselves, and the notion of a point having properties may sound fishy. Note that this is not a criticism of the BST approach but a comment on the scientific image; in straddling two domains, BST has to accept this basic underlying structure as given by current physical theory. But pointillisme, as Butterfield points out, leads to problems already with respect to the interpretation of physical theories, and all the more so with respect to Lebenswelt concepts.

The successful application of scientific theories in everyday technology certainly testifies to the practical possibility of bridging the gap between science and Lebenswelt. In this paper I try to approach that gap theoretically. Ideally I would like to look at the prospects for capturing our Lebenswelt notion of a substance - a thing with properties and capacities-within the formal framework of BST. This in turn would be preparatory work for a fuller theory of agency since agents with their bodies are, finally, also things with properties and capacities. At the present stage of the theory's development, however, I see no hope of arriving at a sufficiently rich notion of a substance directly. I will have some brief comments on that notion below (Section 3), but the bulk of the paper will be devoted to working out some formal aspects

\footnotetext{
${ }^{6}$ For this development, cf. the work of Placek [20, 21] and also Belnap and Szabó [9], Müller and Placek [19], and Müller [16, 17].
} 
of the representation of limited indeterminism in BST, which I consider to be necessary preparatory work for tackling the notion of a substance. ${ }^{7}$

\section{Discrete and Continuous Representations of Limited Indeterminism}

The phrase "limited indeterminism" I take from a short and, as far as I can see, not very well-known paper by Arthur Prior [23] in which he discusses an argument of the 18th century American philosopher and theologian Jonathan Edwards. In the paper Prior shows that it is wrong to spell out indeterminism as "anything whatsoever can happen", and he emphasizes the role of ordinary things in ordinary situations for limiting what is and what isn't possible in an indeterministic setting. Indeterminism has to be thought of as limited indeterminism - indeed the notion of unlimited indeterminism is most probably incoherent. ${ }^{8}$

In one respect, BST already comes with a built-in notion of limited indeterminism that forms part of its technical core. Here I want to make the role of limited indeterminism in BST more explicit by providing an alternative approach to BST that is directly based on specifying indeterminism in a limited and discrete fashion. Heeding Nuel Belnap's advice that we should be proving theorems, in the following I will prove, as my Theorem 1, that there is an alternative "data format" for BST that I think is closer to Lebenswelt applications while still giving access to the technical machinery of BST worked out so far.

\subsection{Rudiments of BST}

BST is formally based on a given partial ordering of possible point-events, $O W=\langle W, \leq\rangle$. Belnap has suggested, usefully, to call such a set "our world", emphasizing the fact that indeterminism and an open future are nothing otherworldly. Furthermore, $O W$ is unified by "suitable external relations" [13, p. 208], viz., by the tempora-causal relation $\leq$, so that it fulfills even Lewis's definition of what a world is.

A crucial step in generalizing branching time to yield the theory of branching space-times was to note that the notion of a history (a complete possible course of events), which is defined as a maximal linear subset in BT, should

\footnotetext{
${ }^{7}$ Let's hope that the fruitful use of promissory footnotes in BST theory continues in this case as well.

${ }^{8}$ Still, that notion, or some vague variant of it, is behind most arguments against a libertarian account of free will: from the compatibilist side, which holds that freedom and determinism go together, one often hears it said that indeterminism can only deliver chance instead of choice. This contrast is faulty, which one realizes once one sees that indeterminism always has to mean the selection of one of a precisely circumscribed, non-random set of options. So much is clear for indeterminism in physics, but the standard seems to shift once agency under indeterminism is considered.
} 
be generalized to an upward directed set. Histories play a decisive role in any branching theory.

Definition 1 (Chain; directed set; history) A set $E \subseteq W$ is linearly ordered or $a$ chain iff for any $e_{1}, e_{2} \in E$, we have $e_{1} \leq e_{2}$ or $e_{2} \leq e_{1}$. A set $E \subseteq W$ is upward directed iff for any $e_{1}, e_{2} \in E$ there is some $e_{3} \in E$ for which $e_{1} \leq e_{3}$ and $e_{2} \leq e_{3}$. A history in $W$ is a maximal upward directed set, i.e., an upward directed set no proper superset of which is directed. (We often use "directed" as short for "upward directed", and we use " $<$ " for the strict order corresponding to $\leq$ but excluding equality.)

By defining histories to be upward directed, one enforces different readings for an upward vs. a downward fork in the ordering. A downward fork (i.e., three possible point events $e_{1}, e_{2}, e_{3}$ for which $e_{1}<e_{3}, e_{2}<e_{3}$, but $e_{1}$ and $e_{2}$ are incomparable) belongs to one history and thus is not to be read modally, but spatio-temporally, in terms of special relativity's notion of space-like separation of $e_{1}$ and $e_{2} \cdot{ }^{9}$ This corresponds to our intuition that the past is ontologically fixed. An upward fork (i.e., three possible point events $e_{1}, e_{2}, e_{3}$ for which $e_{3}<e_{1}, e_{3}<e_{2}$, but $e_{1}$ and $e_{2}$ are incomparable) however allows for two different readings, a spatio-temporal as well as a modal one: if there is some $e_{4}$ for which $e_{1} \leq e_{4}$ and $e_{2} \leq e_{4}$, then the fork belongs to a single history and is thus modally consistent-again, $e_{1}$ and $e_{2}$ are space-like related. If there is no such $e_{4}$, however, then $e_{1}$ and $e_{2}$ belong to different histories and are thus modally separated, meaning that from the point of view of $e_{3}, e_{1}$ and $e_{2}$ belong to two different, incompatible future courses of events.

The following requirements characterize the partial orderings $O W$ that are models of BST, i.e., they give the axioms of BST:

Definition 2 (BST model) A partial ordering $O W=\langle W, \leq\rangle$ is a model of BST iff

1. $\langle W, \leq\rangle$ is a non-empty, dense partial ordering that has no maxima.

2. Every lower bounded chain $C \subseteq W$ has an infimum in $W$.

3. Every upper bounded chain $C \subseteq h, h$ a history, has a supremum in $h$.

4. (Prior Choice Principle). Given two histories $h_{1}, h_{2}$ and a lower bounded chain $C \subseteq h_{1}-h_{2}$, there is some $e \in h_{1} \cap h_{2}$ such that $e<C$ (i.e., $e<e_{C}$ for all $e_{C} \in C$ ) and $e$ is maximal in $h_{1} \cap h_{2}$.

Requirements (1)-(3) enforce a continuous structure of the models. The prior choice principle (4) requires the existence of particular upward forks in line with an intuitive notion of causal explanation: If $C$ occurs in $h_{1}$ but not in $h_{2}$, then there has to be a point $e$ in the causal past of the chain $C$ immediately

\footnotetext{
${ }^{9}$ Two space-time points are space-like separated iff they cannot be compared via the causaltemporal ordering, meaning that they cannot influence one another causally.
} 
after which the two histories come apart. Such a point $e$, maximal in $h_{1} \cap h_{2}$, is called a choice point for $h_{1}$ and $h_{2}$, and we write $h_{1} \perp_{e} h_{2}$. The natural counterpart to two histories splitting at a point is two histories being undivided at a point: $h_{1} \equiv_{e} h_{2}$ iff $e \in h_{1} \cap h_{2}$, but $e$ is not maximal in the intersection (i.e., there is some $e^{\prime} \in h_{1} \cap h_{2}$ s.t. $e<e^{\prime}$ ). It can be shown [1] that " $\equiv$ " is an equivalence relation partitioning the set of histories containing $e, H_{(e)}$, into local alternatives at $e$. We write this partition as $\Pi_{e}$, and the unique member of $\Pi_{e}$ containing some $h \in H_{(e)}$ is written $\Pi_{e}\langle h\rangle$. Based on these definitions, we can define the important notion of a basic transition:

Definition 3 (Basic transition) Given $O W=\langle W, \leq\rangle$, a basic transition is a pair $t_{i}=\left\langle e_{i}, H_{i}\right\rangle$ (also written $t_{i}=e_{i} \longmapsto H_{i}$ ), where $e_{i}$ is a choice point in $O W$ (i.e., an indeterministic point, maximal in the intersection of two or more histories), and $H_{i}$ is one of the immediate possibilities at that $e_{i}$ (i.e., $H_{i} \in \Pi_{e_{i}}$ ).

Thus, for $H \in \Pi_{e}$, the basic transition $\langle e, H\rangle$ or $e \longmapsto H$ captures the concept of initial $e$ having the immediate outcome $H$.

\subsection{The Continuous Nature of BST as a Problem}

By Definition 2(2,3), the partial ordering of BST is required to be continuous, meaning that the cardinality of a model of BST is always (at least) that of the continuum. The way we specify indeterministic scenarios, e.g., in telling stories, is however different: often a finite amount of information is sufficient to specify what is (possibly) going on. From such a perspective, the continuity of BST should be separated into the continuity of space-time (which is required, but can be viewed as a merely potential infinity) together with a discrete means of specifying indeterministic scenarios in space-time..$^{10}$ The question is whether there is something like a "discrete data format for BST". Such a data format would, by the way, not only allow one to connect BST with aspects of Lebenswelt such as the telling of stories, but may also be a prerequisite for making BST computationally tractable and thus, for linking BST with applications in computer science and elsewhere. Additionally, having such a data format, in which the modal aspect of branching is clearly separated from the spatio-temporal aspect of the location of choice points, may help to clarify the nature of BST's branching. This seems important as Earman [11] has raised the worry that BST's branching might amount to branching within a single space-time, showing that such a theory would violate some rather basic requirements of what a space-time theory could be. It will thus be good to

\footnotetext{
${ }^{10}$ The terminology "discrete" is not entirely satisfactory, but I will stick to it for lack of a better term. Typically, a means of specifying indeterministic scenarios before a given continuous spacetime background will be discrete and in fact finite, but I do not wish to exclude from the outset scenarios in which the indeterminsm involved itself is of a dense or continuous nature. Cases of "busy choice" such as discussed in Belnap et al. [8] are certainly not meant to be excluded.
} 
provide a representation for BST models in which the modal branching is clearly separated from the spatio-temporal ordering.

In BT, the theory of branching time out of which BST was developed, the transition from discrete to continuous and back is effected quite easily. Assuming that continuous histories should be isomorphic to the real line, a continuous model of BT has a discrete submodel given by the choice points labeled by the histories these are elements of, and such a discrete structure can in turn easily be embedded in a continuous model. ${ }^{11}$ Three things are helpful here: for one, it seems quite clear that a continuous history should be one that is isomorphic to the real line. Second, the topology of the branching doesn't play any decisive role in BT (cf. McCall [14] for discussion). And third, since histories in BT are linear, in a given discrete set of moments from which to build a continuous BT model, incomparable moments have to belong to different histories.

In BST the situation is more complex. First, there is no totally obvious default for a continuous history, and even though 4-dimensional Minkowski space-time may be a natural candidate, the theory allows for many more continuous histories, even non-isomorphic ones within a single BST model [16]. Thus, there seems to be too large a class of continuous models within which a given discrete "data set" could be embedded. Second, BST is topologically intricate because of the prior choice principle, meaning that in building a continuous structure from given data, care will have to be taken in order to get the branching right. And third, assuming that the discrete data structure for BST will involve a partial ordering, the double reading of the upward fork (modal vs. spatio-temporal) obviously needs attention. An additional challenge is the possibility of so-called modal funny business, for which see below.

\subsection{From BST to BST+SN}

In what follows I will suggest a discrete data structure for BST models that meets the mentioned challenges. I will have to restrict myself to those BST models for which the individual histories are isomorphic-models, that is, within which one and the same space-time canvas is filled in differently in different histories without itself being affected. While this goes against the spirit of general relativity theory, it is well known that BST has difficulties representing that theory anyway, so that this limitation is at least not something specific to the discrete data format for BST. An important class of BST models for which this restriction is unproblematic is the class of Minkowskian branching structures, in which each history is a Minkowski space-time of the same dimension $[15,22,25]$.

\footnotetext{
${ }^{11}$ In calling such a structure discrete we gloss over cases in which the choice points themselves form a dense or continuous set; cf. footnote 10 above.
} 
Technically, my starting point for the continuous theory is not BST in full generality, but BST with space-time locations, called BST $+\mathrm{S}$ in Müller $[16],{ }^{12}$ further restricted by requiring the absence of modal funny business (see below). The resulting theory will be called BST+SN ("N" for "No modal funny business").

Space-time locations can be added to BST by the following requirement, which is slightly adapted from Müller [16]:

Definition 4 (Branching space-times with space-time locations) A triple $\langle W, \leq, S\rangle$ is a model of branching space-times with space-time locations $(B S T+S)$ iff $\langle W, \leq\rangle$ is a model of branching space-times and $S$ is a partition of $W$ such that

1. For each history $h$ in $W$ and for each $s \in S$, the intersection $h \cap s$ contains exactly one element. (Abusing notation, we sometimes identify the singleton $h \cap s=\{e\}$ with its unique element $e$.)

2. $S$ respects the ordering, i.e., for $s, s^{\prime} \in S$ and $h_{1}, h_{2}$ histories, $s \cap h_{1}=s^{\prime} \cap h_{1}$ iff $s \cap h_{2}=s^{\prime} \cap h_{2}$, and the same for ' $<$ ' and for ' $>$ '.

We will write " $S(e)$ " for the (unique) member of $S$ to which $e$ belongs; $S(e)$ specifies the space-time location of $e$. The induced partial ordering $<_{S}$ on $S$ is defined as follows: For $s_{i}, s_{j} \in S$, let $s_{i}<_{S} s_{j}$ iff there is a history $h$ s.t. $s_{i} \cap h=$ $\left\{e_{i}\right\}, s_{j} \cap h=\left\{e_{j}\right\}$, and $e_{i}<e_{j}$. Space-like relatedness in $S$ is then defined in the obvious way, i.e., $s_{i} \operatorname{SLR}_{S} s_{j}$ iff $s_{i} \not s_{s} s_{j}$ and $s_{j} \nless_{s} s_{i}$.

Note that, following usage in physics, "space-time location" implies a (frame-independent) reference to the space-time continuum, not a (framedependent) reference to a place and a time.

The induced partial ordering $<_{S}$ on the non-empty set $S$ is dense and continuous, and $S$ itself is directed with respect to this ordering; the proof of this fact is obvious (simply fix one history and look at the intersection with $S$ ). We spell this out for future reference:

Fact 1 The set $S$ is directed w.r.t. the partial ordering $<_{s}$. Furthermore, $\left\langle S,<_{S}\right\rangle$ is non-empty, dense and has no maximal elements, and every upper bounded chain in $S$ has a supremum in $S$, just as every lower bounded chain has an infimum.

The additional requirement of "no modal funny business" will be discussed in the following section, once the necessary Definition 7 has been introduced.

\footnotetext{
${ }^{12}$ In that paper it is also proved that such models of BST automatically satisfy an additional intuitive postulate about the ordering of suprema in different histories, originally due to Weiner. Cf. Müller [16] for details.
} 


\subsection{Picking the Right Data Format I: Distilling Discrete Structures}

Earlier approaches to Minkowskian branching structures, cited above, also suggested what amounts to a discrete data structure for BST+SN in the special case in which $S$ is isomorphic to Minkowski space-time. These approaches specified what were to be the histories in a resulting continuous model by a pasting construction that relied on the specification of the spatio-temporal location of the choice points for each history, together with the notion of undividedness of the past. These approaches seem to handle situations involving finitely many histories well enough, but the general case remains problematic in a number of ways. ${ }^{13}$

In order to find a more adequate and more transparent discrete representation of BST $+\mathrm{SN}$ models, I will here first look at the way in which one can extract discrete information from a continuous BST + SN model. Belnap [4] has pointed out clearly the importance of basic transitions (Definition 3 above) for BST theory. A first step towards distilling discrete structure from $O W$ is to consider the set of basic transitions in $O W$ :

$$
T R(O W):=\left\{t_{i} \mid t_{i} \text { is a basic transition in } O W\right\} .
$$

There is a sense in which the set $T R(O W)$ may be called "discrete": it contains as elements not all the (continuously many) possible point events of $O W$, but is based on just those "where the action is", i.e., the indeterministic choice points.

In another sense, however, it is misleading to call $T R(O W)$ "discrete". ${ }^{14}$ After all, its elements are basic transitions $t_{i}$ that are represented not just via a discrete, point-like initial, $e_{i}$, but also a set of histories, $H_{i}$-and histories, being maximal directed subsets of $O W$, are continuous. This means that the full continuous history structure of $O W$ is contained in $T R(O W)$, so that it will not be possible to specify something like $T R(O W)$ without first specifying something as "big" as the histories in $O W$ itself. In other words: while $T R(O W)$ does single out a discretized version of $O W$, it cannot be used as a generic discrete data format for BST models because it is internally continuous.

It is possible to distill a truly discrete structure from $\operatorname{TR}(O W)$ via the following consideration: All that is important for a transition is, first, the location of its initial and, second, the compatibility or incompatibility with other transitions, i.e., the information which transitions can occur together in one history. The latter information is very conveniently contained in the set of histories specifying a transition's outcome in the $T R(O W)$ data format $\left(H_{i}\right.$

\footnotetext{
${ }^{13}$ In fact, Müller [15] got a cardinality requirement wrong, cf. Wroński and Placek [25]. The newer papers of Placek and Wroński [22] and Wroński and Placek [25] successfully remove some limitations, but are still faced with the possibility of unwanted histories arising through the pasting construction. Also, I find Condition 6 of Wroński and Placek [25] difficult to grasp intuitively.

${ }^{14}$ Of course, the reservations about the term "discrete" mentioned in footnote 10 apply in any case. The point here at issue is an additional complication that will be remedied.
} 
simply gives one the histories in which $t_{i}$ occurs), but the information can be represented in a leaner fashion. To see this, we define a partial ordering on the set $T R(O W),<_{T}$, as follows (where $t_{i}=\left\langle e_{i}, H_{i}\right\rangle$, and similarly for other subscripts):

$$
t_{i}<_{T} t_{j} \quad \text { iff } e_{i}<e_{j} \text { and } H_{\left(e_{j}\right)} \subseteq H_{i} .
$$

That is, a transition $t_{i}$ precedes the transition $t_{j}$ iff the latter's initial, $e_{j}$, is causally later than that of $t_{i}$ and can occur in the outcome of $t_{i}$. It is obvious that $<_{T}$ is a partial ordering (from the respective properties of $<$ and $\subseteq$ ).

A set of transitions $T R=\left\{t_{i}=\left\langle e_{i}, H_{i}\right\rangle \mid i \in I\right\}$ is called consistent iff there is a history in the intersection of all outcomes, i.e., iff $\cap_{i \in I} H_{i} \neq \emptyset$. We note as a fact that a common upper bound w.r.t. $<_{T}$ secures consistency:

Fact 2 Let $T R=\left\{t_{i}=\left\langle e_{i}, H_{i}\right\rangle \mid i \in I\right\}$ be a set of transitions, and let $t_{k}$ be a transition s.t. $t_{i}<_{T} t_{k}$ for all $i \in I$. Then $T R$ is consistent.

Proof By the definition of the ordering, $H_{\left(e_{k}\right)} \subseteq H_{i}$ for all $i \in I$.

Let us also note as a fact that in $T R(O W)$, no transition has a "lonely" initial:

Fact 3 If $t=\langle e, H\rangle \in T R(O W)$, then there is also some $t^{\prime}=\left\langle e^{\prime}, H^{\prime}\right\rangle \in T, t \neq t^{\prime}$, for which $e=e^{\prime}$.

Proof Basic transitions $t=\langle e, H\rangle$ by definition have an indeterministic initial, i.e., there are at least two $H, H^{\prime} \in \Pi_{e}$. Thus together with $t$, also some $t^{\prime}=$ $\left\langle e, H^{\prime}\right\rangle \in T R(O W)$.

Given the concept of consistency of a set of transitions and the partial order $<_{T}$, we can finally give a succinct definition of the requirement of "no modal funny business" invoked in Section 2.3. The definition given here corresponds to what is called "explanatory funny business" in Müller et al. [18]. First, two preparatory definitions:

Definition 5 (Blatant inconsistency) A set of transitions $T R$ is blatantly inconsistent iff there are $t_{i}=\left\langle e_{i}, H_{i}\right\rangle \in T R$ and $t_{j}=\left\langle e_{j}, H_{j}\right\rangle \in T R, t_{i} \neq t_{j}$, for which $e_{i}=e_{j}$.

Definition 6 ((Proper) downward extension) A set of transitions $T R^{\prime}$ is a downward extension of a set of transitions $T R$ iff (i) $T R \subseteq T R^{\prime}$ and (ii) for all (new) $t_{i} \in T R^{\prime}-T R$ there is (already) some $t_{j} \in T R$ for which $t_{i}<_{T} t_{j}$. A proper downward extension is one for which $T R \subsetneq T R^{\prime}$.

A set of transitions that is blatantly inconsistent contains two incompatible local alternatives from some $\Pi_{e}$. It is thus immediately obvious why such a set of transitions cannot occur in one history: after all, $\Pi_{e}$ partitions the histories 
containing $e$, and partitions do not overlap. A downward extension of a set of transitions can be viewed as the addition of more causal information and thus, as possibly providing an account of why, e.g., some set of transitions is inconsistent. Intuitively, such an account should always be available. This intuition is captured in the following definition of "modal funny business":

Definition 7 (Modal funny business) In a BST-model $O W=\langle W, \leq\rangle$ there is modal funny business if there is an inconsistent set of transitions $T R \subseteq$ $T R(O W)$ such that no downward extension of $T R$ is blatantly inconsistent.

Modal funny business thus means that there are transitions that are inconsistent (that cannot occur together in one history) even though we cannot give an account of that inconsistency in terms of local inconsistency. Such would be the case, e.g., when an outcome ' + ' is possible here, and an outcome ' + ' is possible over there, but there is no history in which ' + ' occurs both here and over there-a modal correlation of a sort that has been accused of involving "spooky action at a distance". ${ }^{15}$ In what follows, working with $\mathrm{BST}+\mathrm{SN}$ models we exclude such cases-certainly not because they are not interesting, but just because it seems prudent to tackle the easier case first.

The partial ordering $<_{T}$, together with the initials' location, turns out to be sufficient to represent the initial BST model $O W$. On our way towards a discrete data structure, we can remove the continuous aspect of $T R(O W)$ (contained in the set of histories specifying the outcome) explicitly by the following maneuver:

Definition 8 (Discrete representation of $O W$ ) Let $O W=\langle W, \leq, S\rangle$ be a $\mathrm{BST}+\mathrm{SN}$ model, let $T R(O W)$ be its set of basic transitions, $<_{T}$ the partial ordering on $T R(O W)$ defined above, and let $I$ be an index set of the cardinality of $T R(O W)$ such that $T R(O W)=\left\{t_{i}=\left\langle e_{i}, H_{i}\right\rangle \mid i \in I\right\}$. Then the quadruple

$$
D(O W):=\left\langle S,<_{S}, T, \prec\right\rangle,
$$

where $<_{S}$ is the partial ordering on $S$ induced by $\leq$,

$$
T:=\left\{\tau_{i}:=\left\langle i, s_{i}\right\rangle \mid i \in I, s_{i}=S\left(e_{i}\right)\right\},
$$

and the partial ordering $\prec$ is defined via

$$
\left\langle i, s_{i}\right\rangle \prec\left\langle j, s_{j}\right\rangle \quad \text { iff } \quad t_{i}<_{T} t_{j}
$$

is called the discrete representation of $O W$.

\footnotetext{
${ }^{15}$ Such a case would also fulfill the original definition of "modal funny business" by Belnap [2, 3]; the definition given here, taken from Müller et al. [18], extends these earlier approaches.

As is well known, quantum mechanics predicts space-like correlations [12], and such correlations have been experimentally verified since the 1980s. It is not clear, however, that modal correlations of the kind discussed here are involved. See also footnote 20 below.
} 
The terminology is adequate: in $D(O W)$, the continuous aspect of $T R(O W)$ has been removed, and a structure like $D(O W)$ can be specified without specifying continuous histories. ${ }^{16}$

\subsection{Picking the Right Data Format II: Discrete Proto-BST+SN-structures}

So far we have shown that from a given BST+SN model one can distill an alternative representation that one can call, perhaps cum grano salis, "discrete". We will now continue by establishing facts about that discrete representation and giving a fully explicit definition of our chosen discrete data format (Definition 10 below).

By the construction, $D(O W)$ fulfills a compatibility requirement for the orderings $<_{S}$ and $\prec$ :

Fact 4 Let $D=D(O W)=\left\langle S,<_{S}, T, \prec\right\rangle$ be derived from a $B S T+S N$-model $O W$ via Definition 8 , and let $\tau_{i}=\left\langle i, s_{i}\right\rangle, \tau_{j}=\left\langle j, s_{j}\right\rangle \in T$ be s.t. $\tau_{i} \prec \tau_{j}$. Then $s_{i}<s s_{j}$.

Proof $\tau_{i}=\left\langle i, s_{i}\right\rangle \in T$ corresponds to $t_{i}=\left\langle e_{i}, H_{i}\right\rangle \in T R(O W)$, and similarly for $\tau_{j}$. By definition, $\tau_{i} \prec \tau_{j}$ iff $t_{i}<_{T} t_{j}$ iff $e_{i}<e_{j}$ and $H_{\left(e_{j}\right)} \subseteq H_{i}$. Since $s_{i}=S\left(e_{i}\right)$ and $s_{j}=S\left(e_{j}\right)$, the claim follows by compatibility of the orderings $<$ and $<_{S}$ (cf. Definition 4 above).

The partial ordering $\langle T, \prec\rangle$ has some further meshing properties that follow from Fact 2. These properties show that a "downward fork" in $T$ has to be read purely spatio-temporally and not modally, thus sustaining the intuition of uniqueness of the past:

Fact 5 Let $D=D(O W)=\left\langle S,<_{S}, T, \prec\right\rangle$ be derived from a $B S T+S N$-model $O W$, and let $\tau_{i}, \tau_{j}, \tau_{k} \in T$ be such that $\tau_{i} \prec \tau_{k}$ and $\tau_{j} \prec \tau_{k}$. Then:

1. If $s_{i}=s_{j}$, then $\tau_{i}=\tau_{j}$. (Uniqueness of past outcomes.)

2. If $s_{i}<s s_{j}$, then $\tau_{i} \prec \tau_{j}$. (Consistency of the past.)

3. If $s_{j}<_{S} s_{i}$, then $\tau_{j} \prec \tau_{i}$. (Consistency of the past.)

4. If $s_{i} \operatorname{SLR}_{S} s_{j}$, then for all $\tau_{l} \in T$ for which both $s_{l}<_{s} s_{i}$ and $s_{l}<_{S} s_{j}$, we have $\tau_{l} \prec \tau_{i}$ iff $\tau_{l} \prec \tau_{j}$. (Consistency of the common past of $S L R$ events.)

Proof By assumption, the $\tau_{*}$ are derived from BST-transitions $t_{*}$, and by Fact $2, t_{i}=\left\langle e_{i}, H_{i}\right\rangle$ and $t_{j}=\left\langle e_{j}, H_{j}\right\rangle$ are consistent since $H_{\left(e_{k}\right)} \subseteq H_{i} \cap H_{j}$. In case (1), by consistency we have $e_{i}=e_{j}$ and also $H_{i}=H_{j}$, thus $t_{i}=t_{j}$, giving

\footnotetext{
${ }^{16}$ Of course, the space-time background $S$ will be continuous. What is important is that the specification of an indeterministic scenario happening before such a background does not additionally introduce a continuous amount of information, as was the case with $T R(O W)$.- Again we gloss over the possibility that the transitions in $T$ themselves might form a dense or continuous set.
} 
rise to $i=j$ and thus $\tau_{i}=\tau_{j}$. In case (2), consistency gives $e_{i}<e_{j}$ and again $H_{\left(e_{j}\right)} \subseteq H_{i}$, which yields $t_{i}<_{T} t_{j}$ and therefore $\tau_{i} \prec \tau_{j}$. Case (3) works in he same way. In case (4), assume that there is $t_{l}<_{T} t_{i}$ for which $s_{l}<_{S} s_{i}$ and $s_{l}<_{S} s_{j}$ (the argument for $t_{l}<_{T} t_{j}$ works the same way). Pick some history $h \in H_{\left(e_{k}\right)}$; we have $e_{i}, e_{j}, e_{l} \in h$ and thus, via $s_{l}<_{S} s_{j}$, also $e_{l}<e_{j}$. To establish that $t_{l}<_{T} t_{j}$ and thus $\tau_{l} \prec \tau_{j}$, we need to show that $H_{\left(e_{j}\right)} \subseteq H_{l}$. From $t_{l}<_{T} t_{j}<_{T} t_{k}$ we get $h \in H_{l} \cap H_{\left(e_{j}\right)}$, so $H_{l}=\Pi_{e_{l}}\langle h\rangle=\left\{h^{\prime} \mid h^{\prime} \equiv_{e_{l}} h\right\}$. Now let $h^{\prime} \in H_{\left(e_{j}\right)}$; as $e_{l} \leq e_{j}$, we have $h^{\prime} \equiv_{e_{l}} h$ and thus $h^{\prime} \in H_{l}$.

Just given the information contained in the partial ordering $\langle T, \prec\rangle$, we can already reconstruct the important relations of initial-equivalence and spacelike relatedness via the following definition.

Definition 9 Let $\langle T, \prec\rangle$ be a partial ordering of transitions, and let $\tau_{i}=$ $\left\langle i, s_{i}\right\rangle, \tau_{j}=\left\langle j, s_{j}\right\rangle \in T$. We define:

1. $\tau_{i}$ and $\tau_{j}$ are initial-equivalent, $\tau_{i}$ IE $\tau_{j}$, iff $s_{i}=s_{j}$ and for all $\tau_{k} \in T, \tau_{k} \prec \tau_{i}$ iff $\tau_{k} \prec \tau_{j}$.

2. $\tau_{i}$ and $\tau_{j}$ are space-like related, $\tau_{i} \operatorname{SLR} \tau_{j}$, iff $s_{i} \operatorname{SLR}_{S} s_{j}$ and for all $\tau_{k}=$ $\left\langle k, s_{k}\right\rangle \in T$ for which $s_{k}<_{S} s_{i}$ and $s_{k}<_{S} s_{j}, \tau_{k} \prec \tau_{i}$ iff $\tau_{k} \prec \tau_{j}$.

The idea behind these definitions is to read the modal structure out of the partial ordering $\prec$ on $T$. As to (1), the fact that two transitions $\tau_{i}$ and $\tau_{j}$ from $T$ have initials located at the same spatio-temporal position $\left(s_{i}=s_{j}\right)$ of itself does not prove that these initials should correspond to one and the same possible point event in a corresponding BST model. If however the past indeterministic happenings do not give a reason for modal differentiation, then the initials are equal as possible point events. A similar motivation lies behind definition (2). Two possible point events in a BST model are space-like related if they are order-incompatible, but nonetheless members of one and the same history. Again, space-like relatedness of the positions of the initials $\left(s_{i} \operatorname{SLR}_{S} s_{j}\right)$ is not enough: there must be no transition in the common past differentiating the initials either, i.e., the initials have to be SLR as possible point events. ${ }^{17}$

In the following lemma, clauses (1) and (2) show that for a discrete structure derived from a BST model, Definition 9 in fact captures the corresponding BST relations. Clauses (3)-(5) note some further useful facts that follow from the prior choice principle together with the absence of modal funny business;

\footnotetext{
${ }^{17}$ The notion of space-like relatedness suggests modal compatibility. By extending this notion from point events to transitions, we rely on the absence of modal funny business: on that assumption the space-like relatedness of initials of two basic transitions is enough to guarantee the existence of a history in which both transitions occur. The simplest cases of modal funny business involve space-like related initials but modally incompatible transitions from these initials; cf. footnote 15 above and the discussion in Belnap [2].
} 
clause (3) captures the possibility of downward extension that is noted in Müller et al. [18].

Lemma 1 Let $O W$ be a $B S T+S N$ model and $D(O W)=\left\langle S,<_{S}, T, \prec\right\rangle$ the corresponding discrete representation. Then for $\tau_{i}=\left\langle i, s_{i}\right\rangle$ and $\tau_{j}=\left\langle j, s_{j}\right\rangle$ corresponding to BST-transitions $t_{i}=\left\langle e_{i}, H_{i}\right\rangle$ and $t_{j}=\left\langle e_{j}, H_{j}\right\rangle$, we have

1. $\tau_{i} \mathrm{IE} \tau_{j}$ iff $e_{i}=e_{j}$. (Thus, no transition in $T$ is initial-lonely, by Fact 3.)

2. $\tau_{i} \operatorname{SLR} \tau_{j}$ iff $e_{i} \operatorname{SLR} e_{j}$.

3. If $s_{i}<_{S} s_{j}$ but $\tau_{i} \nprec \tau_{j}$, then there is a pair of previous transitions $\tau_{k} \neq \tau_{l}$ s.t. $\tau_{k}$ IE $\tau_{l}$ that splits off $\tau_{i}$ from $\tau_{j}: \tau_{k} \preceq \tau_{i}$ and $\tau_{l} \prec \tau_{j}$ (and thus, $\tau_{k} \nprec \tau_{j}$ and $\left.\tau_{l} \nprec \tau_{i}\right)$.

4. If $s_{i} \operatorname{SLR}_{S} s_{j}$ but not $\tau_{i} \operatorname{SLR} \tau_{j}$, then there is a pair of previous transitions $\tau_{k} \neq \tau_{l}$ s.t. $\tau_{k}$ IE $\tau_{l}$ that splits off $\tau_{i}$ from $\tau_{j}: \tau_{k} \prec \tau_{i}$ and $\tau_{l} \prec \tau_{j}$ (and thus, $\tau_{k} \nprec \tau_{j}$ and $\left.\tau_{l} \nprec \tau_{i}\right)$.

5. Let $U_{i}, U_{j} \subseteq T$ be maximally consistent, and let $C \subseteq S$ be a lower bounded chain in $S$; set $s_{\perp}=\inf C$. If for all $s \in C$ there are $\tau_{i}=\left\langle i, s_{i}\right\rangle \in U_{i}-U_{j}$ or $\tau_{j}=\left\langle j, s_{j}\right\rangle \in U_{j}-U_{i}$ s.t. $s_{i}<_{s} s\left(s_{j}<_{s} s\right)$, then there are also $\tau_{k}=\left\langle k, s_{k}\right\rangle \in$ $U_{i}$ and $\tau_{l}=\left\langle l, s_{l}\right\rangle \in U_{j}$ s.t. $\tau_{k} \neq \tau_{l}, \tau_{k}$ IE $\tau_{l}$, and $s_{k}=s_{l} \leq_{s} s_{\perp}$.

Proof

(1) “ $\Leftarrow ":$ Given $e_{i}=e_{j}$, clause (1) of Definition 9 follows directly.

" $\Rightarrow$ ": Let $e_{i} \neq e_{j}$ and $s\left(e_{i}\right)=s\left(e_{j}\right)$; by uniqueness of history-intersections with $S$ there is no history containing both $e_{i}$ and $e_{j}$. By the prior choice principle there is some $t=\langle e, H\rangle$ in $O W$ with $e<e_{i}$ and $t<_{T} t_{i}, t \nless_{T} t_{j}$. This $t$ corresponds to a $\tau \in T$ separating $\tau_{i}$ from $\tau_{j}$.

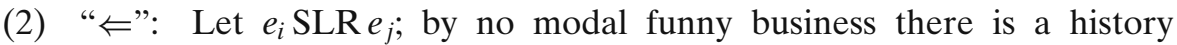
$h \in H_{i} \cap H_{j}$. Let $\tau_{k}=\left\langle k, s_{k}\right\rangle \in T$ with $s_{k}<_{S} s_{i}$ and $s_{k}<_{S} s_{j}$ be one of the transitions relevant for clause (2) of Definition 9; $\tau_{k}$ corresponds to $t=\langle e, H\rangle$, a transition in $O W$ for which $s(e)=s_{k}$. If $h \in H$ then $e<e_{i}$ and $e<e_{j}$ and in fact $t<_{T} t_{i}$ and $t<_{T} t_{j}$. If, on the other hand, $h \notin H$, then neither $t<_{T} t_{i}$ nor $t<_{T} t_{j}$. The corresponding facts about $\tau_{i}, \tau_{j}$ and $\tau_{k}$ follow directly.

" $\Rightarrow$ ": Let $e_{i}$ and $e_{j}$ be such that $s\left(e_{i}\right) \operatorname{SLR}_{S} s\left(e_{j}\right)$ but not $e_{i} \operatorname{SLR} e_{j}$; this means that there is no history containing both $e_{i}$ and $e_{j}$. Similarly to $(1 " \Rightarrow$ "), by the prior choice principle and no modal funny business there is a transition $t=\langle e, H\rangle$ with initial $e$ in the common past of $e_{i}$ and $e_{j}$ that separates the two; this $t$ corresponds to a $\tau \in T$ separating $\tau_{i}$ from $\tau_{j}$.

(3) The antecedent translates as $H_{i} \cap H_{\left(e_{j}\right)}=\emptyset$. Let $h_{i} \in H_{i}$ and $h_{j} \in H_{j} \subseteq$ $H_{\left(e_{j}\right)}$. We distinguish two cases:

(i) $h_{i} \perp_{e_{i}} h_{j}$. We can derive the required $\tau_{k}$ and $\tau_{l}$ from $t_{k}=t_{i}$ and $t_{l}=$ $e_{i} \longmapsto \Pi_{\left(e_{i}\right)}\left\langle h_{j}\right\rangle$. 
(ii) Otherwise the prior choice principle for $e_{i} \in h_{i}-h_{j}$, together with the requirement of no modal funny business, delivers the required pair of transitions.

(4) The definition of SLR on $T$ already gives one distinguishing transition. Similarly to (3), the prior choice principle together with no modal funny business gives the required pair of transitions.

(5) This condition captures the topological content of the prior choice principle. $U_{i}$ and $U_{j}$ correspond to maximally consistent sets of transitions from $T R(O W)$, where consistency means that all outcomes share a history. Let $h_{i}$ and $h_{j}$ be respective histories. As all histories are isomorphic to $S$, the chain $C$ in $S$ has an isomorphic image $C_{i}$ in $h_{i}$. The antecedent then means that no element of $C_{i}$ is in $h_{j}$, i.e., $C_{i} \subseteq h_{i}-h_{j}$. Let $e_{\perp}=\inf C_{i}$; by isomorphy of histories we have $S\left(e_{\perp}\right)=s_{\perp}$. The prior choice principle assures us of a choice point in the past of $C_{i}$, i.e., there is some $e \in h_{i} \cap h_{j}$ for which $e<C_{i}$ and which is maximal in $h_{i} \cap h_{j}$. As $e \leq e_{\perp}$ ( $e_{\perp}$ being the infimum of $C_{i}$ ), we have $S(e) \leq s_{\perp}$, and we can choose $\tau_{k}$ and $\tau_{l}$ to be the $T$-representatives of $t_{k}=e \longmapsto \Pi_{e}\left\langle h_{i}\right\rangle$ and $t_{l}=e \longmapsto \Pi_{e}\left\langle h_{j}\right\rangle$.

In the following we will be working with a discrete data structure that could be given as some $D(O W)$, but also independently, e.g., via some story we might tell about indeterministic happenings. This data structure thus forms the interface between BST's technical machinery and our Lebenswelt concepts. The motivation for the following definition is therefore twofold: It describes structures such as can be distilled from a given BST+SN model (cf. Fact 6 below), and it describes structures that we can specify in everyday terms. In full detail, we define the notion of a discrete proto-BST+SN-structure as follows:

Definition 10 (Discrete proto-BST $+\mathrm{SN}$-structure) A quadruple $D=\left\langle S,<_{S}\right.$, $T, \prec\rangle$ is called a discrete proto-BST+SN-structure iff

1. $\langle S,<S\rangle$ is a non-empty, continuous, upward directed partial ordering without maxima;

2. $\langle T, \prec\rangle$ is a partial ordering, the elements of $T$ have the form $\tau_{i}=\left\langle i, s_{i}\right\rangle$ with $s_{i} \in S$, and $T$ is left-unique (i.e., if $\left\langle i, s_{i}\right\rangle,\left\langle j, s_{j}\right\rangle \in T$ with $i=j$, then $s_{i}=s_{j}$ as well);

3. if $\tau_{i} \prec \tau_{j}$, then $s_{i}<_{S} s_{j}$;

4. $T$ fulfills the uniqueness of the past requirements mentioned in Fact 5: For $\tau_{i}, \tau_{j}, \tau_{k} \in T$ such that $\tau_{i} \prec \tau_{k}$ and $\tau_{j} \prec \tau_{k}$, the following conditions hold:

(a) if $s_{i}=s_{j}$, then $\tau_{i}=\tau_{j}$;

(b) if $s_{i}<s s_{j}$, then $\tau_{i} \prec \tau_{j}$;

(c) if $s_{j}<_{S} s_{i}$, then $\tau_{j} \prec \tau_{i}$;

(d) if $s_{i} \operatorname{SLR}_{S} s_{j}$, then for all $\tau_{l} \in T$ for which both $s_{l}<_{s} s_{i}$ and $s_{l}<_{S} s_{j}$, we have $\tau_{l} \prec \tau_{i}$ iff $\tau_{l} \prec \tau_{j}$; 
5. $\quad T$ fulfills the prior choice principle related requirements noted in Fact 3 and in Lemma 1(3-5): For $\tau_{i}, \tau_{j} \in T$,

(a) $\tau_{i}$ is not initial-lonely, i.e., there is some $\tau_{k} \in T, \tau_{i} \neq \tau_{k}$, for which $\tau_{i}$ IE $\tau_{k}$;

(b) if $s_{i}<_{S} s_{j}$ but $\tau_{i} \nprec \tau_{j}$, then there is a pair of previous transitions $\tau_{k} \neq \tau_{l}$ s.t. $\tau_{k}$ IE $\tau_{l}, \tau_{k} \preceq \tau_{i}$ and $\tau_{l} \prec \tau_{j}$;

(c) if $s_{i} \operatorname{SLR}_{S} s_{j}$ but not $\tau_{i} \operatorname{SLR} \tau_{j}$, then there is a pair of previous transitions $\tau_{k} \neq \tau_{l}$ s.t. $\tau_{k}$ IE $\tau_{l}, \tau_{k} \prec \tau_{i}$ and $\tau_{l} \prec \tau_{j}$;

(d) if $U_{i}, U_{j} \subseteq T$ are maximally consistent and $C \subseteq S$ is a lower bounded chain in $S$ with $s_{\perp}=\inf C$, and if for all $s \in C$ there are $\tau_{i}=\left\langle i, s_{i}\right\rangle \in$ $U_{i}-U_{j}$ or $\tau_{j}=\left\langle j, s_{j}\right\rangle \in U_{j}-U_{i}$ s.t. $s_{i}<_{s} s\left(s_{j}<_{s} s\right)$, then there are also $\tau_{k}=\left\langle k, s_{k}\right\rangle \in U_{i}$ and $\tau_{l}=\left\langle l, s_{l}\right\rangle \in U_{j}$ s.t. $\tau_{k} \neq \tau_{l}, \tau_{k}$ IE $\tau_{l}$, and $s_{k}=$ $s_{l} \leq S s_{\perp}$.

We note as a fact that any $D(O W)$ derived from a BST $+\mathrm{SN}$-model $O W$ indeed fulfills this definition:

Fact 6 Let $O W$ be a $B S T+S N$ model, and let $D(O W)$ be the corresponding discrete representation. Then $D(O W)$ is a discrete proto-BST-structure.

Proof The proof is obvious, given (1) Fact 1, (2) the construction of $D(O W)$, (3) Fact 4, (4) Lemma 5 and (5) Fact 3 and Lemma 1.

Conditions (1) and (2) allow us, conveniently, to use the set $I:=\left\{i \mid\left\langle i, s_{i}\right\rangle \in\right.$ $T\}$ as an index set for $T$, and (3) is a precondition for interpreting the ordering $\prec$ on $T$ in the sense of BST. (4) is required for assuring that the given partial ordering can be interpreted in terms of a consistent past, and (5) assures the presence of causal accounts of "why this rather than that" and the absence of modal funny business.

Fact 6 provides ample motivation for this choice of data format from the formal point of view of BST theory. We cannot state a motivation for the definition from the Lebenswelt side with equal rigor, but the following should be noted: (1), which ensures that the resulting BST+SN model will be continuous and without maxima, arguably captures our concept of space-time, ${ }^{18}$ (2) is just a technical convenience, and (3) is needed for a causal interpretation of transitions in space-time. ${ }^{19}$ As to (4), (a)-(d) are indeed expressions of the

\footnotetext{
${ }^{18}$ This is of course a tricky business. Since-I would argue-we have to start with separate Lebenswelt concepts of space and time, space-time is a theoretical notion. Not all space-time models in the theoretical physics literature satisfy the partial order and the directedness requirement. (E.g., space-times with causal loops fail to be partially ordered.) For present purposes we can state that it is a known desideratum of the future development of BST theory in general to allow for such extensions. At the present state of development, the requirements seem reasonable because they allow us to make at least some progress, and the Minkowski space-time of special relativity as well as many space-times of general relativity do fulfill the requirements.

${ }^{19}$ Again, worries about backward causation may crop up; cf. the previous note.
} 
intuition that "... is in the past of ..." is a transitive relation, and that the past of any event is unique. (5) cashes out three requirements of the causal completeness of stories: (a) Alternatives are alternatives to something else, and in a complete story we need to be told to what, so there can be no initiallonely initials. (b, c) If transitions are not compatible, we want to be told why that is so, and an answer to that question consists in pointing out a pair of simultaneous local alternatives such that one enables the one transition and the other, the other. (d) In line with the prior choice principle, "giving reasons" should also extend to chains. ${ }^{20}$

\subsection{From the Discrete Data Structure Back to BST+SN}

In order to show that our discrete representation, a discrete proto-BST+SNstructure according to Definition 10, is in fact an adequate data format for $\mathrm{BST}+\mathrm{SN}$, we will now show two things. First, we will show that such a representation $D$ allows one to construct a (continuous) BST $+\mathrm{SN}$ model $C(D)$. Secondly, we will show that if $D=D(O W)$ was derived from a BST+SN model $O W$, the resulting continuous model $C(D(O W))$ will be isomorphic to the initial model $O W$ in the sense of (a) being based on the same space-time background and (b) showing the same structure of basic transitions.

Before we state and prove the adequacy of our discrete data format (Theorem 1 below), there is some preliminary work to be done, as we wish to identify histories with consistent scenarios. Extending the considerations leading to Definition 9, we can define when a set of transitions is consistent in the sense of not containing a reason for why it shouldn't occur in one history. ${ }^{21}$

Definition 11 (Consistency) A set $U \subseteq T$ of transitions is called consistent iff for any $\tau_{i}, \tau_{j} \in U$, one of the following holds:

1. $\tau_{i} \mathrm{IE} \tau_{j}$ and $i=j$ (i.e., $\tau_{i}=\tau_{j}$ ) or

2. $\tau_{i} \prec \tau_{j}$ or

3. $\tau_{j} \prec \tau_{i}$ or

4. $\tau_{i} \operatorname{SLR} \tau_{j}$.

\footnotetext{
${ }^{20}$ Again there is a fine point, connected with the notion of modal funny business (cf. footnote 15 above): Requiring (5) rules out modal funny business, while there seem to be quantum-mechanical cases that do exhibit such funny business. One way out here is to say that empirically, all that can be established is vanishing probabilities of outcomes, not modal impossibility, so that any story involving modal funny business can also be told as a story involving just probabilistic funny business. While this may be a feeble reply (I think it is), the technical challenges of working out the fully general case demand that the simpler case involving no funny business treated here should be worked out first. Yapf. (Yet another promissory footnote.)

${ }^{21}$ For this definition to make good sense we also require the absence of modal funny business; cf. the facts about what is there called "combinatorial funny business" in Müller et al. [18].
} 
Alternatively, we can write this definition explicitly in terms of the four possible cases for the spatio-temporal relation of initials, which we state as a fact (the proof is obvious):

Fact $7 A$ set $U \subseteq T$ of transitions is consistent iff for any $\tau_{i}=\left\langle i, s_{i}\right\rangle, \tau_{j}=$ $\left\langle j, s_{j}\right\rangle \in U$, the following holds:

1. if $s_{i}=s_{j}$ then $\tau_{i}=\tau_{j}$, and

2. if $s_{i}<s s_{j}$ then $\tau_{i} \prec \tau_{j}$ and

3. if $s_{j}<_{S} s_{i}$ then $\tau_{j} \prec \tau_{i}$ and

4. if $s_{i} \operatorname{SLR}_{S} s_{j}$ then for all $\tau_{k}$ with $s_{k}<_{s} s_{i}$ and $s_{k}<_{S} s_{j}$, we have $\tau_{k} \prec \tau_{i}$ iff $\tau_{k} \prec \tau_{j}$.

Based on this definition and the requirements on $T$ spelled out in Definition 10, we can prove that downward extensions in $T$ retain consistency:

Lemma 2 Let $U \subseteq T$ be consistent, let $\tau_{i} \in U$, and let $\tau_{j} \in T$ be such that $\tau_{j} \prec \tau_{i}$. Then the set $U \cup\left\{\tau_{j}\right\}$ is also consistent.

Proof Let $\tau_{k} \in U$. We prove that the requirements of Fact 7 hold for the pair $\left(\tau_{j}, \tau_{k}\right)$, depending on the spatio-temporal relation of their initials $s_{j}$ and $s_{k}$ :

(1) $s_{j}=s_{k}$. We have $\tau_{j} \prec \tau_{i}$ and (by consistency of $U$ ) also $\tau_{k} \prec \tau_{i}$, so that $\tau_{j}=\tau_{k}$ follows by clause 4(a) of Definition 10 .

(2) $s_{j}<s s_{k}$. Here we differentiate by the relation between $s_{k}$ and $s_{i}$ :

(a) $s_{k}=s_{i}$ : By consistency, $\tau_{k}=\tau_{i}$, so $\tau_{j} \prec \tau_{k}$.

(b) $s_{k}<_{S} s_{i}$ : Similarly to (1), we have $\tau_{j} \prec \tau_{i}$ and (by consistency of $U$ ) also $\tau_{k} \prec \tau_{i}$, so that $\tau_{j} \prec \tau_{k}$ follows by clause $4(\mathrm{~b})$.

(c) $s_{i}<_{S} s_{k}$ : By consistency, $\tau_{i} \prec \tau_{k}$, and thus by transitivity of $\prec, \tau_{j} \prec \tau_{k}$.

(d) $s_{i} \mathrm{SLR}_{S} s_{k}$ : By consistency, $\tau_{i} \operatorname{SLR} \tau_{k}$, and $s_{j}$ is in the common past of $s_{i}$ and $s_{k}$, so that by clause $4(\mathrm{~d})$, together with $\tau_{j} \prec \tau_{i}$ we also have $\tau_{j} \prec \tau_{k}$.

(3) $s_{k}<_{S} s_{j}$. Similarly to (1), we have $\tau_{j} \prec \tau_{i}$ and (by consistency of $U$ ) also $\tau_{k} \prec \tau_{i}$, so that $\tau_{k} \prec \tau_{j}$ follows by clause $4(\mathrm{~b})$.

(4) $s_{j} \mathrm{SLR}_{s} s_{k}$. Again we have to consider the relation between $s_{k}$ and $s_{i}$ :

(a) $s_{k}=s_{i}$ : This cannot be since $s_{j}<_{s} s_{i}$ by assumption.

(b) $s_{k}<_{S} s_{i}$ : As in 2(b) above.

(c) $s_{i}<_{S} s_{k}$ : By transitivity of $<_{S}$, this cannot be since $s_{j}<_{S} s_{i}$.

(d) $s_{i} \operatorname{SLR}_{S} s_{k}$ : By consistency of $U, \tau_{i} \operatorname{SLR} \tau_{k}$.

Assume that it is not the case that $\tau_{j} \operatorname{SLR} \tau_{k}$. Then there has to be some $\tau_{l}$ for which $s_{l}<_{S} s_{j}, s_{l}<_{S} s_{k}, \tau_{l} \prec \tau_{j}$ but $\tau_{l} \nprec \tau_{k}$ (the other case is symmetrical). By transitivity of $\prec, \tau_{l} \prec \tau_{i}$, contradicting $\tau_{i} \operatorname{SLR} \tau_{k}$.

The following obvious corollary will be useful later on: 
Corollary 1 Let $U \subseteq T$ be maximally consistent, $\tau_{i} \in U$, and $\tau_{j} \prec \tau_{i}$. Then $\tau_{j} \in U$.

A certain mirror image of this corollary expressing one aspect of no modal funny business will also be helpful:

Lemma 3 Let $U \subseteq T$ be maximally consistent and let $\tau_{i} \in T-U$ (i.e., $U \cup\left\{\tau_{i}\right\}$ is inconsistent). Then there is (already) some $\tau_{j} \in U$ for which the pair $\left(\tau_{i}, \tau_{j}\right)$ is inconsistent and for which $s_{j} \leq_{S} s_{i}$.

Proof As $U \cup\left\{\tau_{i}\right\}$ is inconsistent, there has to be some $\tau_{l} \in U$ such that $\left(\tau_{i}, \tau_{l}\right)$ is an inconsistent pair. We will find the required $\tau_{j} \in U$ by considering the four cases for the spatio-temporal relation between $s_{i}$ and $s_{l}$ :

(1) $s_{i}=s_{l}$. We can take $\tau_{j}=\tau_{l}$.

(2) $s_{l}<_{S} s_{i}$. Again, $\tau_{j}=\tau_{l}$ will do.

(3) $s_{i}<S s_{l}$. By requirement 5(b) of Definition 10 there is some $\tau_{j} \in T$ for which $\tau_{j} \prec \tau_{l}$ but $\tau_{j} \nprec \tau_{i}$; by the above Corollary, $\tau_{j} \in U$.

(4) $s_{i} \mathrm{SLR}_{S} s_{l}$. Requirement 5(c) of Definition 10 gives some $\tau_{j} \in T$ for which $\tau_{j} \prec \tau_{l}$ but $\tau_{j} \nprec \tau_{i}$; again by the Corollary, $\tau_{j} \in U$.

The following lemma shows that the requirements on $T$ spelled out in Definition 10 are, as intended, strong enough to guarantee that there can be no modal funny business.

Lemma 4 (Absence of explanatory funny business) Let $U \subseteq T$ be inconsistent. Then there is a downward extension $U^{\prime} \supseteq U$ (i.e., for $\tau_{k} \in U^{\prime}-U$ there is already some $\tau_{i} \in U$ with $\tau_{k} \prec \tau_{i}$ ) s.t. $U^{\prime}$ is blatantly inconsistent (i.e., $U^{\prime}$ contains $\tau_{i} \neq \tau_{j}$ s.t. $\left.\tau_{i} \mathrm{IE} \tau_{j}\right)$.

Proof Let $\tau_{i}=\left\langle i, s_{i}\right\rangle, \tau_{j}=\left\langle j, s_{j}\right\rangle \in U$ be such that the pair $\left(\tau_{i}, \tau_{j}\right)$ is inconsistent. We have to find a downward extension $U^{\prime}$ of $U$ that is blatantly inconsistent. In fact we will show that there are $\tau_{k}, \tau_{l} \in T$ for which $\tau_{k} \neq \tau_{l}$, $\tau_{k} \mathrm{IE} \tau_{l}$, and $\tau_{k} \preceq \tau_{i}, \tau_{l} \preceq \tau_{j}$; we can then set $U^{\prime}=U \cup\left\{\tau_{k}, \tau_{l}\right\}$. As in Lemma 3 we distinguish four cases depending on the spatio-temporal relation between $s_{i}$ and $s_{l}$ :

(1) $s_{i}=s_{j}$. We can take $\tau_{k}=\tau_{i}, \tau_{l}=\tau_{j}$.

(2) $s_{i}<s s_{j}$. Clause 5(b) of Definition 10 guarantees the existence of the required $\tau_{k}, \tau_{l}$.

(3) $s_{j}<_{S} s_{i}$. This case works exactly like (2).

(4) $s_{i} \operatorname{SLR}_{S} s_{l}$. Clause 5(c) of Definition 10 guarantees the existence of the required $\tau_{k}, \tau_{l}$.

We now state and prove our main theorem. 
Theorem 1 Let $D=\left\langle S,<_{S}, T, \prec\right\rangle$ be a discrete proto-BST+SN-structure. Then the triple $C(D)=\langle W, \leq, \Sigma\rangle$, defined below, is a model of $B S T+S N$ whose set of basic transitions, $T R(C(D))$, is isomorphic to $\langle T, \prec\rangle$.

Proof The idea behind the construction of a continuous BST + SN model from $D$ is to read off the modal information from $T$, using the maximal consistent subsets of $T$ to determine which histories there are. By choosing the representatives of possible point events in the model appropriately, the pasting together of these histories is effected automatically. We define:

Definition 12 Given a discrete proto-BST $+\mathrm{SN}$-structure $D=\left\langle S,<_{S}, T, \prec\right\rangle$, the corresponding continuous model is the triple $C(D)=\langle W, \leq, \Sigma\rangle$, where the set $\Sigma$ and the partial ordering $\langle W, \leq\rangle$ are constructed as follows:

- Let $\mathfrak{C}$ be the set of maximal consistent subsets of $T$. For each $U \in \mathfrak{C}$, let the corresponding history representative be

$$
h_{U}:=\left\{\langle s, V\rangle \mid s \in S, V=\left\{\tau_{k}=\left\langle k, s_{k}\right\rangle \in U \mid s_{k}<_{S} s\right\} .\right.
$$

- Define $W$ to be the union of all these histories, i.e.,

$$
W:=\cup_{U \in \mathfrak{C}} h_{U} .
$$

- Define the ordering $\leq$ via the spatio-temporal ordering together with the notion of the uniqueness of the past, i.e.,

$$
\langle s, V\rangle \leq\left\langle s^{\prime}, V^{\prime}\right\rangle \quad \text { iff } \quad s \leq s s^{\prime} \text { and } V=\left\{\tau_{k} \in V^{\prime} \mid s_{k}<s s\right\} .
$$

The corresponding strict ordering (excluding equality) will be denoted "<".

- The set $\Sigma$ is lifted from $S$ in the obvious way:

$$
\Sigma:=\{\{\langle s, V\rangle \in W\} \mid s \in S\}
$$

Obviously $W$ is a non-empty set (as $S \neq \emptyset$ ), and $\leq$, being based on $<_{S}$, is a partial ordering on $W$. It now remains to prove that $C(D)$ is indeed a BST $+\mathrm{SN}$ model, and that its set of basic transitions, $T R(C(D))$, is isomorphic to $D$ 's transition ordering $\langle T, \prec\rangle$.

In order to prove anything about the basic transitions in $C(D)$, or indeed the prior choice principle, we need to determine the histories in $C(D)$, i.e., the maximal directed sets. We establish that the $h_{U}$ are indeed the histories in $\langle W, \leq\rangle$, treating the two directions as the separate Lemmas 5 and 6 . We will then establish the BST properties via Lemma 8 and finally prove the claimed isomorphism via Lemma 9.

Before we start with these lemmas, we note the following useful fact:

Fact 8 Let $g \subseteq W$ be directed, and let $\langle s, V\rangle,\left\langle s, V^{\prime}\right\rangle \in g$. Then $V=V^{\prime}$. (I.e., in a directed set in $W$, no space-time point $s \in S$ can occur with two different labels.) 
Proof Assume that $g$ is directed and that there are $e=\langle s, V\rangle, e^{\prime}=\left\langle s, V^{\prime}\right\rangle \in g$. By directedness, there has to be some $e^{*}=\left\langle s^{*}, V^{*}\right\rangle$ s.t. $e \leq e^{*}$ and $e^{\prime} \leq e^{*}$. By definition of the ordering, this means that $V=\left\{\tau_{k} \in V^{*} \mid s_{k}<_{S} s\right\}=V^{\prime}$.

Lemma 5 The sets $h_{U}, U \in \mathfrak{C}$, are maximal directed sets in $\langle W, \leq\rangle$.

Proof Let $U \in \mathfrak{C}$ be a maximal consistent set of transitions from $T$, and let $h_{U}$ be the set of pairs $\langle s, V\rangle$ defined above. We need to show that $h_{U}$ is (a) directed and (b) maximally so.

(a) Let $e_{i}=\left\langle s_{i}, V_{i}\right\rangle \in h_{U}, i=1,2$. We have to find some $e^{*} \in h_{u}$ s.t. $e_{1} \leq e^{*}$ and $e_{2} \leq e^{*}$. Now by directedness of $S$ there is some $s^{*} \in S$ for which $s_{i}<_{S} s^{*}, i=1,2$. Choosing $e^{*}=\left\langle s^{*},\left\{\tau_{k} \in U \mid s_{k}<_{S} s^{*}\right\}\right\rangle$, the required ordering relations hold by definition.

(b) In order to prove maximality, let $e=\langle s, V\rangle \in W-h_{U}$ be a point extending $h_{U}$. By definition, $h_{U}$ already contains a point $\left\langle s, V_{U}\right\rangle$, and by Fact 8 , if $h_{U} \cup\{e\}$ were directed, we would have to have $V=V_{U}$, contradicting the assumption that $e \notin h_{U}$. So no proper extension of $h_{U}$ is directed.

Lemma 6 Let $h$ be a maximal directed set in $\langle W, \leq\rangle$. Then there is some $U \in \mathfrak{C}$ (i.e., a maximal consistent set of transitions from $T$ ) such that

$$
h=h_{U} .
$$

Proof Let $h$ be a maximal directed subset of $W$; we know that its members are of the form $\left\langle s, V_{s}\right\rangle$ with $s \in S$ and $V_{s} \subseteq T$, where by Fact 8 , the $V_{s}$ are unique for each $s$. By the definition of $W$, for $\tau_{k}=\left\langle k, s_{k}\right\rangle \in V_{s}$ we have $s_{k}<_{s} s$.

Let

$$
T_{h}:=\cup_{\left\langle s, V_{s}\right\rangle \in h} V_{s}
$$

be the union of all transition labels occurring in $h$, so that $T_{h} \subseteq T$.

We prove the lemma in four steps (a)-(d).

(a) $T_{h}$ is a consistent set of transitions.

Proof Assume otherwise, i.e., assume that there are $\tau_{1}, \tau_{2} \in T_{h}$ violating Definition 11. By construction of $T_{h}$ this means that there have to be $e_{i}=\left\langle s_{i}, V_{i}\right\rangle \in h$ such that $\tau_{i} \in V_{i}, i=1,2$. By directedness of $h$ there also has to be some $e^{*}=\left\langle s^{*}, V^{*}\right\rangle \in h$ with $e_{1} \leq e^{*}$ and $e_{2} \leq e^{*}$. By definition of the ordering this means that $V_{1} \subseteq V^{*}$ and $V_{2} \subseteq V^{*}$, so that $\left\{\tau_{1}, \tau_{2}\right\} \subseteq V^{*}$, meaning that $V^{*}$ is inconsistent. But as $e^{*} \in h \subseteq W$, we also have $e^{*} \in h_{U}$ for some maximal consistent set of transitions $U \in \mathfrak{C}$, so that $V^{*} \subseteq U$ has to be consistent.

(b) For $e=\left\langle s, V_{s}\right\rangle \in h$, we have

$$
V_{s}=\left\{\tau_{k} \in T_{h} \mid s_{k}<_{S} s\right\}
$$


Proof " $\subseteq$ " follows by definition of $T_{h}$ together with the definition of $W$. For " $\supseteq$ ", let $\tau_{k} \in T_{h}$ with $s_{k}<_{S} s$. By the definition of $T_{h}$, there has to be some $e^{\prime}=\left\langle s^{\prime}, V^{\prime}\right\rangle \in h$ with $\tau_{k} \in V^{\prime}$, and by directedness of $h$, there is some $e^{*}=\left\langle s^{*}, V^{*}\right\rangle \in h$ for which $e \leq e^{*}$ and $e^{\prime} \leq e^{*}$. By the definition of the ordering, $V^{\prime} \subseteq V^{*}$ so that $\tau_{k} \in V^{*}$, and also by that definition, $\tau_{k} \in V_{s}$.

(c) For each $s \in S$ there is some $\left\langle s, V_{s}\right\rangle \in h$.

Proof Let

$$
S_{h}:=\left\{s \in S \mid \text { there is some }\left\langle s, V_{s}\right\rangle \in h\right\}
$$

be the space-time points present in $h$. Our aim is to establish $S_{h}=S$.

(c.i) $S_{h}$ is downward closed, i.e., if $s \in S_{h}$ and $s^{\prime}<_{s} s$ then also $s^{\prime} \in S_{h}$. To show this, let $s \in S_{h}$, i.e., there is $e=\left\langle s, V_{s}\right\rangle \in h$; by definition of $W, e \in h_{U}$ for some $U \in \mathfrak{C}$. Let $s^{\prime} \in S$ be such that $s^{\prime}<_{S} s$. Setting $V^{\prime}=\left\{\tau_{i} \in V_{s} \mid s_{i}<s s^{\prime}\right\}$, the point $e^{\prime}=\left\langle s^{\prime}, V^{\prime}\right\rangle \in h_{U} \subseteq W$ and $e^{\prime}<e$. Clearly $h \cup\left\{e^{\prime}\right\}$ is directed: given $e^{\prime \prime} \in h$, by directedness of $h$ there exists a common upper bound $e^{*} \in h$ for $e$ and $e^{\prime \prime}$, and since $e^{\prime}<e$, that $e^{*}$ is also a common upper bound for $e^{\prime}$ and $e^{\prime \prime}$. So if we had $s^{\prime} \notin S_{h}$, then $e^{\prime}$ would properly extend $h$ as a directed set, contradicting the assumption that $h$ is maximally directed.

(c.ii) If there is some $\tau_{k} \in T-T_{h}$ such that $T_{h} \cup\left\{\tau_{k}\right\}$ is consistent, then there can be no $s \in S_{h}$ for which $s_{k}<_{S} s$. To show this, assume otherwise, so that there is some such $\tau_{k}$ and some $e=\left\langle s, V_{s}\right\rangle \in h$ with $s_{k}<_{S} s$. As $\tau_{k} \notin T_{h}$, we have $\tau_{k} \notin V_{s}$. As $e \in h \subseteq W$, we must have $e \in h_{U}$ for some maximally consistent $U \in \mathfrak{C}$, and $V_{s}=\left\{\tau_{i} \in\right.$ $\left.U \mid s_{i}<_{s} s\right\}$. As $\tau_{k} \notin V_{s}$, by maximality of $U, U \cup\left\{\tau_{k}\right\}$ must be inconsistent. Now by Lemma 3 this means that there is already some $\tau_{j} \in U$ with $s_{j} \leq s s_{k}$ and such that $\left(\tau_{j}, \tau_{k}\right)$ are inconsistent. But as $s_{j} \leq_{S} s_{k}<_{S} s$, we must have $\tau_{j} \in V_{s}$ and thus $\tau_{j} \in T_{h}$, meaning that $T_{h} \cup\left\{\tau_{k}\right\}$ cannot be consistent.

(c.iii) Let $S^{\prime}=S-S_{h}$ be the complement of $S_{h}$. If $S^{\prime}=\emptyset$ then (c) is established. Otherwise, select some maximally consistent set of transitions $U \in \mathfrak{C}$ for which $T_{h} \subseteq U$. We will show that the proper extension of $h$,

$$
h^{*}:=h \cup\left\{\langle s, V\rangle \mid s \in S^{\prime}, V=\left\{\tau_{k} \in U \mid s_{k}<s\right\}\right\} \subseteq W
$$

is also directed, contradicting the maximality of $h$ and thus proving (c).

In order to show that $h^{*}$ is directed, let $e_{i}=\left\langle s_{i}, V_{i}\right\rangle \in h^{*}, i=$ 1,2 . We distinguish three cases.

1. $e_{1}, e_{2} \in h$. A common upper bound is already in $h$ (and thus in $\left.h^{*}\right)$, by directedness of $h$. 
2. $e_{1}, e_{2} \in h^{*}-h$. By directedness of $S$, there is some $s^{*} \in S$ for which $s_{1}<s s^{*}$ and $s_{2}<s s^{*}$. We have $s^{*} \in S^{\prime}$ by (c.i). Let $e^{*}=$ $\left\langle s^{*},\left\{\tau_{k} \in U \mid s_{k}<s^{*}\right\}\right\rangle \in h^{*}$. By the definition of the ordering, $e^{*}$ is a common upper bound for $e_{1}$ and $e_{2}$.

3. $e_{1} \in h, e_{2} \in h^{*}-h$ (the case with 1 and 2 reversed is symmetrical). Again by directedness of $S$, there is some $s^{*} \in S$ for which $s_{1}<_{S} s^{*}$ and $s_{2}<_{S} s^{*}$, and again by (c.i), $s^{*} \in S^{\prime}$. Let $V^{*}=$ $\left\{\tau_{k} \in U \mid s_{k}<s^{*}\right\}$ and $e^{*}=\left\langle s^{*}, V^{*}\right\rangle \in h^{*}$. By the definition of the ordering, $e_{2} \leq e^{*}$. In order to show that also $e_{1} \leq e^{*}$, we need to show that $V_{1}=\left\{\tau_{k} \in V^{*} \mid s_{k}<_{S} s_{1}\right\}$. By (b) we know that $V_{1}=\left\{\tau_{k} \in T_{h} \mid s_{k}<_{S} s_{1}\right\}$, so " $\subseteq$ " holds via $T_{h} \subseteq U$. Assume that there is some $\tau_{k} \in V^{*}$ for which $\tau_{k} \notin T_{h}$ but $s_{k}<_{S} s_{1} . \tau_{k} \in V^{*} \subseteq U$ means that $T_{h} \cup\left\{\tau_{k}\right\}$ is consistent; but then by (c.ii) we couldn't have $s_{1} \in S_{h}$. So " $\supseteq$ " holds as well, and we have established that $e^{*}$ is a common upper bound for $e_{1}$ and $e_{2}$.

(d) We can now proceed to show that $T_{h}$ is maximally consistent and thus a member of $\mathfrak{C}$-once that is proved, by (b) and (c) we have $h=h_{T_{h}}$, and the lemma is proved.

Thus, assume that $T_{h}$ is not maximally consistent, so that for some $\tau_{k} \in$ $T-T_{h}, T_{h} \cup\left\{\tau_{k}\right\}$ is consistent. By (c.ii) this implies that there can be no $s \in S_{h}$ for which $s_{k}<_{S} s$. But there is some $s \in S$ for which $s_{k}<_{S} s$, and by (c) we have established that $S_{h}=S$, so $s \in S_{h}$, a contradiction. Therefore, $T_{h}$ must be maximally consistent.

This concludes the proof of Lemma 6.

Having identified the histories in $C(D)$, we now need to establish the BST axioms. The ones connected with the ordering properties can easily be lifted from corresponding facts about $S$; the more difficult bit is to establish the prior choice principle. It is useful to establish the following lemma first, which proves that our construction in fact gives back the desired choice points:

Lemma 7 Let $h_{1}=h_{U_{1}}$ and $h_{2}=h_{U_{2}}$ be histories in $C(D), U_{1}, U_{2} \in \mathfrak{C}$, and let $e=\left\langle s_{\perp}, V\right\rangle \in h_{1} \cap h_{2}$. Then $h_{1} \perp_{e} h_{2}$ iff there are $\tau_{k} \in U_{1}, \tau_{l} \in U_{2}$ such that $\tau_{k} \neq \tau_{l}, \tau_{k}$ IE $\tau_{l}$, and $s_{k}=s_{l}=s_{\perp}$.

\section{Proof}

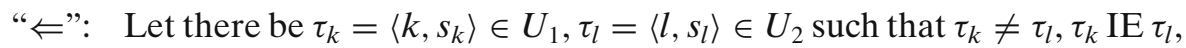
and $s_{k}=s_{l}=s_{\perp}$. We have to show that $e$ is maximal in $h_{1} \cap h_{2}$. So let $e^{\prime}=\left\langle s^{\prime}, V^{\prime}\right\rangle \in h_{1}$ s.t. $e<e^{\prime}$. By definition, $V^{\prime}=\left\{\tau_{i} \in U_{1} \mid s_{i}<s^{\prime}\right\}$. Thus, $\tau_{k} \in V^{\prime}$. But $\tau_{l} \notin V^{\prime}$ because $\left(\tau_{k}, \tau_{l}\right)$ is an inconsistent pair and $V^{\prime} \subseteq U_{1}$ is consistent. So $e^{\prime} \notin h_{2}$, and $e$ is indeed maximal in in $h_{1} \cap h_{2}$.

" $\Rightarrow$ ": Let $h_{1} \perp_{e} h_{2}$, i.e., let $e=\left\langle s_{\perp}, V_{\perp}\right\rangle$ be maximal in $h_{1} \cap h_{2}$. Let $C$ be a chain in $S$ s.t. $s_{\perp}=\inf C$ but $s_{\perp} \notin C$. We can show that the 
antecedent of Definition 10(5d) is fulfilled: Let $s \in C$ (so that $s_{\perp}<_{S} s$ ). Let $V_{1}=\left\{\tau_{m} \in U_{1} \mid s_{m}<_{s} s\right\}$ and $V_{2}=\left\{\tau_{m} \in U_{2} \mid s_{m}<_{s} s\right\}$. We have $e_{1}=\left\langle s, V_{1}\right\rangle \in h_{1}$, but as $e<e_{1}$ and $e$ is maximal in $h_{1} \cap h_{2}$, we have $e_{2}=$ $\left\langle s, V_{2}\right\rangle \in h_{2}-h_{1}$, so $V_{1} \neq V_{2}$. This means that there is some $\tau_{i} \in U_{i}-$ $U_{j}$ with $s_{i}<_{S} s$ or some $\tau_{j} \in U_{j}-U_{i}$ with $s_{j}<_{S} s$. So Definition $10(5 \mathrm{~d})$ applies and gives us $\tau_{k}=\left\langle k, s_{k}\right\rangle \in U_{1}$ and $\tau_{l}=\left\langle l, s_{l}\right\rangle \in U_{2}$ for which $\tau_{k} \neq \tau_{l}, \tau_{k}$ IE $\tau_{l}$, and $s_{k}=s_{l} \leq s s_{\perp}$. Since $e \in h_{1} \cap h_{2}$, the case $s_{k}<_{s} s_{\perp}$ is excluded, leaving $s_{k}=s_{l}=s_{\perp}$.

By Lemma 7 we have identified the choice points and thus, the indeterministic structure of $C(D)$ : the lemma says that a point $e$ is a choice point, maximal in the intersection of two histories $h_{1}$ and $h_{2}$, iff the two histories are differentiated by transitions $\tau_{k}$ and $\tau_{l}$ with that point $e$ as initial.

We can now formulate the $\mathrm{BST}+\mathrm{SN}$ requirements as a lemma:

Lemma 8 For $C(D)=\langle W, \leq, \Sigma\rangle$ derived from a discrete proto-BST+SNstructure $D$, the following holds:

1. $\langle W, \leq\rangle$ is a non-empty, dense partial order that has no maxima.

2. Every lower bounded chain $C \subseteq W$ has an infimum in $W$.

3. Every upper bounded chain $C \subseteq h$, h a history, has a supremum in $h$.

4. (Prior Choice Principle). Given two histories $h_{1}, h_{2}$ and a lower bounded chain $C \subseteq h_{1}-h_{2}$, there is some $e \in h_{1} \cap h_{2}$ such that $e<C$ (i.e., $e<e_{C}$ for all $\left.e_{c} \in C\right)$ and $e$ is maximal in $h_{1} \cap h_{2}$.

5. (Existence of space-time location). $\Sigma$ fulfills the requirements on spatiotemporal location laid out in Definition 4.

6. (No modal funny business). There is no modal funny business according to Definition 7.

Proof

(1)-(3) are obvious, working in a selected history and noting the respective properties of $S$.

(4) The proof is exactly parallel to the proof of Lemma $7(" \Rightarrow ")$.

(5) Existence and uniqueness of the intersection of $\Sigma$ with a history follow directly from Lemma 6(c) and Fact 8, respectively. Preservation of the ordering holds by the construction of $\Sigma$.

(6) This follows directly from Lemma 4, given the identification of basic transitions in $C(D)$ laid out in Lemma 7.

This concludes the proof of Lemma 8.

As the final step in proving our main Theorem 1, it remains to be shown that the given transition structure of $D$ is in fact isomorphic to the derived transition structure of $C(D)$. 
Lemma 9 Let $T R=T R(C(D))$ be the set of basic transitions of the continuous $B S T+S N$ structure $C(D)=\langle W, \leq, \Sigma\rangle$ derived from the given discrete proto$B S T+S N$-structure $D=\left\langle S,<_{S}, T, \prec\right\rangle$, and let $<_{T}$ be the transition ordering on $T R$. Then $\left\langle T R,<_{T}\right\rangle$ and $\langle T, \prec\rangle$ are isomorphic, i.e., there is a bijection $f$ between $T$ and $T R$ s.t. for $\tau_{i}=\left\langle i, s_{i}\right\rangle, \tau_{j}=\left\langle j, s_{j}\right\rangle \in T$ we have:

1. (Preservation of initial location) Let $\tau_{i}=\left\langle i, s_{i}\right\rangle$ and $f\left(\tau_{i}\right)=e_{i} \longmapsto H_{i}$. Then we have $\Sigma\left(e_{i}\right)=s_{i}$.

2. (Preservation of ordering) $\tau_{i} \prec \tau_{j}$ iff $f\left(\tau_{i}\right)<_{T} f\left(\tau_{j}\right)$.

Proof Let $\tau_{k}=\left\langle k, s_{k}\right\rangle \in T$; by Definition 10(5a), there is some $\tau_{l}=\left\langle l, s_{l}\right\rangle \in T$ s.t. $\tau_{k} \neq \tau_{l}$ and $\tau_{k}$ IE $\tau_{l}$. There are $U_{1}, U_{2} \in \mathfrak{C}$ for which $\tau_{k} \in U_{1}, \tau_{l} \in U_{2}$. Let $V=\left\{\tau_{m} \in U_{1} \mid s_{m}<_{S} s_{k}\right\}$; we have $e=\left\langle s_{k}, V\right\rangle \in h_{U_{1}} \cap h_{U_{2}}$. By Lemma 7, $e$ is maximal in this intersection, so that $t_{k}:=e \longmapsto \Pi_{e}\left\langle h_{U_{1}}\right\rangle$ is a basic transition: $t_{k} \in$ $T R$. We set $f\left(\tau_{k}\right)=t_{k}$. Note that this definition is independent of the choice of $\tau_{l}$ and of the sets $U_{1}, U_{2}$, by the fact that $\Pi_{e}$ partitions the set of histories containing $e$. Claim (1) follows by construction, as $\Sigma(e)=s_{k}{ }^{22}$

In order to prove that $f$ is bijective, we show that it injective and surjective. For injectiveness, let $\tau_{i}, \tau_{j} \in T$ be such that $f\left(\tau_{i}\right)=e_{i} \longmapsto H_{i}=e_{j} \longmapsto H_{j}=$ $f\left(\tau_{j}\right)$. From $e_{i}=e_{j}$ we immediately get $\tau_{i} \operatorname{IE} \tau_{j}$, and remembering that $\Pi_{e_{i}}$ is a partition, we then also have $\tau_{i}=\tau_{j}$. For surjectiveness, let $t_{i}=e_{i} \longmapsto H_{i} \in T R$, so that $e_{i}$ is maximal in some $h_{1} \cap h_{2}$ with $h_{1} \in H_{i}$; this $h_{1}=h_{U}$ for some $U \in \mathfrak{C}$. By Lemma 7 there are $\tau_{k} \neq \tau_{l} \in T$ s.t. $\tau_{k}$ IE $\tau_{l}$ and $\tau_{k} \in U$; plugging this into the definition of $f$ shows that in fact $f\left(\tau_{k}\right)=t_{i}$.

Finally, as to the ordering (2), let $\tau_{i}, \tau_{j} \in T$ s.t. $\tau_{i} \prec \tau_{j}$. This is a consistent pair, so that there is some $U \in \mathfrak{C}$ containing them both; for $t_{i}=e_{i} \longmapsto H_{i}=f\left(\tau_{i}\right)$ and $t_{j}=e_{j} \longmapsto H_{j}=f\left(\tau_{j}\right)$ we have $h_{U} \in H_{i} \cap H_{j}$, so that $e_{i}<e_{j}$. By the fact that $\Pi_{e_{i}}$ is a partition we also get $H_{\left(e_{j}\right)} \subseteq H_{i}$ and thus, $t_{i}<_{T} t_{j}$. In the other direction, let $t_{i}=e_{i} \longmapsto H_{i}, t_{j}=e_{j} \longmapsto H_{j} \in T R$ be s.t. $t_{i}<_{T} t_{j}$, and let $f\left(\tau_{i}\right)=t_{i}$, $f\left(\tau_{j}\right)=t_{j}$. Select some $h \in H_{i} \cap H_{j}$; this has to be $h_{U}$ for some $U \in \mathfrak{C}$. Thus $\tau_{i}, \tau_{j} \in U$, and their spatio-temporal relation together with the consistency of $U$ gives us $\tau_{i} \prec \tau_{j}$.

This concludes the proof of the main Theorem 1: the continuous structure of $\mathrm{BST}+\mathrm{SN}$ and the discrete proto-BST $+\mathrm{SN}$-structures of Definition 10 have been shown to characterize the same class of indeterministic scenarios.

\section{A Brief Note on Substances in BST}

BST is based on a single ontological category: that of a possible point event, where "event" is used in the sense that has become prominent in space-time

\footnotetext{
${ }^{22}$ In writing this equation we have in fact carried the abuse of notation one step further than announced in Definition 4. Lemma 8(5) ensures that no harm can result.
} 
theories: a point-like, spatio-temporally located entity that may have, e.g., field strengths as its properties. In normal usage, "event" is used differently: it is reserved for a happening involving some ordinary thing, or substance; a change or, more broadly, a transition involving the thing. And this is just one way in which substances are important; they enter into many of our basic Lebenswelt concepts, most notably, agency.

If one wishes to stay close to the formalism of BST-which is a sensible thing to do, given that BST is the best formal framework for indeterminism out there-, then the challenge is to find a way of representing ordinary things and the changes they can undergo within the given, sparse ontological framework, in which there are no things-there isn't even stuff. Can one build up, so to speak, an ordinary thing out of BST's possible point events? A first approximation is to idealize an ordinary thing as something that has no extension: a point-like thing that only has location, no width or breadth or height, nor orientation.

Belnap has shown how to represent such idealized things within the framework of BST. His own approach to agency within BST [6] works with a, literally, thin notion of an agent: a set of point events such that the intersection with each history is a (point-thin) world line. While this means falling prey to anorexia philosophica, it has the great value of being relativistically sound. And idealizing things to be point-thin is a common strategy in physics; it is present, e.g., in the treatment of heavenly bodies through most of the history of astronomy, and it is useful for many other applications as well. As long as one is far enough away, real size doesn't matter, only position does.

Improving on such thin representations is difficult: our Lebenswelt concept of a rigid body has a hard time given relativity theory. As is well known, the notion of a rigid body, i.e., of an extended something that is unanimously wholly present at any single time of its existence, appears to break down in a relativistic setting for rather elementary reasons. An extended thing wholly present at a time would seem to have to be at rest with respect to itself, but that would seem to mean that it has to determine a preferred rest frame, which is anathema to what people take to be the spirit of relativity theory. Spelled out in another way, a rigid body, when pushed from the left, would have to move its right boundary instantaneously. I guess that is how we really think about the things we deal with in ordinary life. But such behavior is impossible given relativity theory's limitation on the speed of signal transmission. The problem is really complicated, and there is no solution to it that could claim to be universally accepted.

I wish to propose two ways of thinking about extended things in BST that might turn out to be helpful, maybe in combination. One is to embed one of these relativity-friendly, point-thin things within an ordinary thing: Given that we have to represent a thing in a BST model with the help of a set of possible point events, we can single out a point, one may think of it as the "center" of the thing (this may or may not be the center of gravity, such as is common for the representation of heavenly bodies), whose motion is represented via a point-thin branching tree as in Belnap's construction. This point-thin idealized 
thing provides for a unique and uncontroversial rest frame for the extended thing at each moment of its existence: the hyperplane orthogonal to the tangent to the worldline of the "center" defines a natural rest frame for the whole object. ${ }^{23}$ This approach has a certain artificiality to it, and one worry (raised in discussion by Mark Brown, who traces it back to Chisholm) is that we may seem to be postulating necessary parts, which would indeed be a bad thing. I guess a reply to this may be that for practical purposes one can prove that it doesn't matter which point one selects, but this would certainly have to be worked out in some detail. A second way of thinking about extended things is to go for another extreme and say that all that really matters when one thinks about causation and the capacities of things is which indeterministic transitions a thing initiates. In the end this may lead one to represent a thing via a set of basic transitions, rather than via a set of possible point events. When thinking about agency, this move may have the benefit of capturing the notion of attribution of an action that is at the center of the free will debate: a thing, and thus an agent, will be defined via the transitions that can be attributed to it. The spatio-temporal extension of a thing would, on this view, be seen as secondary, filling the space between the points at which the action is.

This may sound like a tall story. I think it is, but I also think that bringing together science and Lebenswelt in a respectable theory of indeterminism is supremely difficult. I am looking forward to Nuel Belnap's next move.

\section{Conclusions}

Branching space-times is the best formal theory of indeterminism out there. Besides aiming at the formal elucidation of the concept of indeterminism, BST is also an attempt at bridging the gap between science and Lebenswelt. Ultimately this will have to mean that central Lebenswelt concepts such as agency can be captured in BST, or in some suitable extension of BST that retains the initial scientific respectability.

I have argued that capturing agency involves at least three steps: from BST (or BST+SN) to a discrete data format for limited indeterminism; from that format to the implementations of substances and their capacities, and from BST enriched with substances and capacities to agency as a capacity of substantive agents. In this paper the first step has been taken, as witnessed by Theorem 1; I have also supplied some sketchy remarks about the second step. My hope would be that the present development can also help to bring BST closer to applications in mainstream metaphysics.

\footnotetext{
${ }^{23}$ If the thing's state of motion changes, these hyperplanes may intersect.
} 
Acknowledgements I would like to thank Anil Gupta for getting the Nuelfest off the ground and for inviting me to give a talk there; thanks are also due to the other local organizers. I would also like to thank my audience for helpful and illuminating discussions; I have immensely benefitted from all the exchanges that this event has made possible through its relaxed and friendly atmosphere. Thanks to Tomasz Placek and to an anonymous referee for helpful comments on a previous draft. Support by the Deutsche Forschungsgemeinschaft is gratefully acknowledged.

Open Access This article is distributed under the terms of the Creative Commons Attribution Noncommercial License which permits any noncommercial use, distribution, and reproduction in any medium, provided the original author(s) and source are credited.

\section{References}

1. Belnap, N. (1992). Branching space-time. Synthese, 92, 385-434.

2. Belnap, N. (2002). EPR-like "funny business" in the theory of branching space-times. In T. Placek, \& J. Butterfield (Eds.), Non-locality and modality (pp. 293-315). Dordrecht: Kluwer.

3. Belnap, N. (2003). No-common-cause EPR-like funny business in branching space-times. Philosophical Studies, 114, 199-221.

4. Belnap, N. (2005). A theory of causation: Causae causantes (originating causes) as inus conditions in branching space-times. British Journal for the Philosophy of Science, 56, 221253.

5. Belnap, N. (2007). From Newtonian determinism to branching-space-time indeterminism. In T. Müller (Ed.), Logik, Begriffe, Prinzipien des Handelns (pp. 18-36). Paderborn: Mentis.

6. Belnap, N. (2010). Prolegomena to norms in branching space-times. Journal of Applied Logic (forthcoming).

7. Belnap, N., \& Perloff, M. (1988). Seeing to it that: A canonical form for agentives. Theoria, 54(3), 175-199.

8. Belnap, N., Perloff, M., \& Xu, M. (2001). Facing the future. Agents and choices in our indeterminist world. Oxford: Oxford University Press.

9. Belnap, N., \& Szabó, L. (1996). Branching space-time analysis of the GHZ theorem. Foundations of Physics, 26(8), 989-1002.

10. Butterfield, J. (2006). Against pointillisme about mechanics. British Journal for the Philosophy of Science, 57(4), 709-753.

11. Earman, J. (2008). Pruning some branches from branching space-times. In D. Dieks (Ed.), The ontology of spacetime II (pp. 187-205). Amsterdam: Elsevier.

12. Einstein, A., Podolsky, B., \& Rosen, N. (1935). Can quantum-mechanical description of physical reality be considered complete? Physical Review, 47(10), 777-780.

13. Lewis, D. (1986). On the plurality of worlds. Oxford: Blackwell.

14. McCall, S. (1990). Choice trees. In J. Dunn, \& A. Gupta (Eds.), Truth or consequences. Essays in honor of Nuel Belnap (pp. 231-244). Dordrecht: Kluwer.

15. Müller, T. (2002). Branching space-time, modal logic and the counterfactual conditional. In T. Placek, \& J. Butterfield (Eds.), Non-locality and modality (pp. 273-291). Dordrecht: Kluwer.

16. Müller, T. (2005). Probability theory and causation: A branching space-times analysis. British Journal for the Philosophy of Science, 56, 487-520.

17. Müller, T. (2007). Branch dependence in the "consistent histories" approach to quantum mechanics. Foundations of Physics, 37(2), 253-276.

18. Müller, T., Belnap, N., \& Kishida, K. (2008). Funny business in branching space-times: Infinite modal correlations. Synthese, 164, 141-159.

19. Müller, T., \& Placek, T. (2001). Against a minimalist reading of Bell's theorem: Lessons from fine. Synthese, 128, 343-379.

20. Placek, T. (2000). Stochastic outcomes in branching space-times. British Journal for the Philosophy of Science, 51, 445-475. 
21. Placek, T. (2010). On propensity-frequentist models for stochastic phenomena with applications to Bell's theorem. In T. Czarnecki, K. Kijania-Placek, V. Kukushkina, \& J. Woleński (Eds.), The analytic way. Proceedings of the 6th European congress on analytic philosophy. London: College Publications.

22. Placek, T., \& Wroński, L. (2009). On infinite EPR-like correlations. Synthese, 167, 1-32.

23. Prior, A. N. (1968). Papers on time and tense. Chapter 6: Limited indeterminism (pp. 59-65). Oxford: Oxford University Press.

24. Sellars, W. (1962). Philosophy and the scientific image of man. In R. Colodny (Ed.), Frontiers of science and philosophy (pp. 35-78). Pittsburgh: University of Pittsburgh Press.

25. Wroński, L., \& Placek, T. (2009). On Minkowskian branching structures. Studies in History and Philosophy of Modern Physics, 40, 251-258. 